\title{
Modelado e implementación de un sistema distribuido para la simulación de reacciones químicas
}

\section{Modeling and implementation of a distributed system for the simulation of chemical reactions}

\author{
MACÍAS-BRAMBILA, Hassem Rubén ${ }^{1} \dagger^{*}$, PULIDO-GONZÁLEZ, Héctor ${ }^{2}$, GÓMEZ-LÓPEZ, \\ Daniela $^{2}$ y GUTIÉRREZ-ÁLVAREZ, Francisco Ramón ${ }^{2}$ \\ ${ }^{1}$ Universidad Tecnológica de Jalisco - División de Mecatrónica, Luis J. Jiménez No. 577, colonia Primero de mayo, C.P. \\ 44979, Guadalajara, Jalisco, México \\ ${ }^{2}$ Universidad de Guadalajara - Centro Universitario de Ciencias Exactas e Ingenierías, Blvd. Gral. Marcelino García \\ Barragán No. 1421, colonia Olímpica, C.P. 44430, Guadalajara, Jalisco, México
}

ID 1 ${ }^{\text {er }}$ Autor: Hassem Rubén, Macías -Brambila / ORC ID: 0000-0002-6540-7464, CVU CONACYT ID: 902812

ID $1^{\text {er }}$ Coautor: Héctor, Pulido-González / ORC ID: 0000-0002-8619-3012, CVU CONACYT ID: 313575

ID $2^{\text {do }}$ Coautor: Daniela, Gómez-López / ORC ID: 0000-0002-0748-9211, CVU CONACYT ID: 967451

ID $3^{\text {er }}$ Coautor: Francisco Ramón, Gutiérrez-Álvarez / ORC ID: 0000-0001-5677-7845, CVU CONACYT ID: 967370

DOI: 10.35429/JEA.2019.21.6.1.9

Recibido: 19 de Agosto, 2019; Aceptado 30 de Noviembre, 2019

\begin{abstract}
Resumen
El presente artículo describe el proceso de análisis, diseño e implementación de un sistema distribuido conformado de una suite de aplicaciones y de dos servidores que realizan el procesamiento para el reconocimiento óptico de patrones, la simulación en 2D y 3D, la gestión de sesiones y además brindan la información de la tabla periódica. Este esquema distribuido establece el marco de referencia para el modelado, representación y simulación de reacciones químicas a través de compuestos y elementos de la tabla periódica, para el apoyo a las actividades presenciales en los laboratorios de química de las Instituciones de Educación que ofertan estas asignaturas. El desarrollo del software requirió de la implementación de la metodología ágil SCRUM para la producción de la suite, la que permitirá a los usuarios a través de las simulaciones la comprensión y entendimiento sobre la interacción de los elementos entre sí, brindando un apoyo visual fundamental para la comprensión de tópicos como los electrones de valencia y los enlaces químicos, lo que permite abonar al desarrollo de las competencias descritas en los perfiles de egreso de dichas Instituciones.
\end{abstract}

Sistema distribuido, Simulador, Reacciones Químicas

\begin{abstract}
This article describes the process of analysis, design and implementation of a distributed system consisting of a suite of applications, in which there is a web, a desktop and a mobile and two servers that perform the processing for the optical recognition of patterns, 2D and 3D simulation, session management and also provide the information of the periodic table. This distributed scheme establishes the frame of reference for the modeling, representation and simulation of chemical reactions through compounds and elements of the periodic table, for the support of classroom activities in the chemistry laboratories of the Educational Institutions that offer these subjects. The development of the software required the implementation of the agile SCRUM methodology for the production of the suite, which will help users through simulations to understand and understand the interaction of the elements with each other, providing visual support fundamental for the understanding of topics such as valence electrons and chemical bonds, which allows the development of the skills described in the graduation competencies of these Institutions.
\end{abstract}

Distributed system, Simulator, Chemical Reactions

Citación: MACÍAS-BRAMBILA, Hassem Rubén, PULIDO-GONZÁLEZ, Héctor, GÓMEZ-LÓPEZ, Daniela y GUTIÉRREZ-ÁLVAREZ, Francisco Ramón. Modelado e implementación de un sistema distribuido para la simulación de reacciones químicas. Revista de Aplicaciones de la Ingeniería. 2019. 6-21: 1-9

\footnotetext{
* Correspondencia del Autor (Correo electrónico: hmacias@utj.edu.mx)

$\dagger$ Investigador contribuyendo como primer autor.
} 


\section{Introducción}

Desde sus comienzos, la humanidad ha sentido curiosidad y fascinación por el mundo que le rodea, tanto por su composición como por los fenómenos que en él se llevan a cabo. Inicialmente, el hombre se valía de explicaciones rudimentarias para instruir sobre los eventos que tenían lugar en su entorno, con el pasar de las épocas y conforme su conocimiento, fue posible entender de mejor forma cómo estaba conformado el mundo y así dar mejores explicaciones a los fenómenos de los que se era testigo día a día. Se logró conocer que la materia estaba conformada por menores unidades, diferentes entre sí, a las que se les llamó elementos, y que eran las combinaciones de éstos las que constituían todo lo que los rodeaba. Dicho principio sigue vigente aún en nuestros días y constituye uno de los pilares fundamentales de lo que hoy conocemos como una de las ciencias de mayor relevancia; la química.

Eventualmente las reacciones químicas ganaron importancia dado que comprender cómo es que se lograba una reacción, permitía obtener determinados resultados que después podían ser aprovechados, lo que representó un gran avance que benefició a una gran cantidad de sectores, de los cuales, aún en nuestros días podemos disfrutar.

En la diversidad de áreas de investigación científica una de las más conocidas, es sin lugar a duda las reacciones químicas, considerando que la materia que se encuentra en el planeta tiene como unidad básica elementos químicos que son complementados entre sí, formando distintos compuestos, ya sea de origen natural cuando se encuentran en abundancia en el medio ambiente o bien, sintéticos cuando tienen un origen humano.

La resolución de reacciones químicas tiende a ser un proceso complicado, e incluso, puede llegar a ser confuso al tratar con reacciones que involucran muchos elementos, así como difícil de imaginar el fascinante comportamiento que tienen las reacciones químicas a nivel molecular. Por otro lado, las herramientas computacionales para estos fines, tales como "Chemical Predictor", resultan ser insuficientes para esta labor, dado que se enfocan en un sólo tipo de reacciones, además de presentar representaciones poco interactivas.
Por lo que se inició con el desarrollo e implementación de una suite de aplicaciones informáticas conformadas de módulos que permiten resolver escenarios como los antes mencionados, y contar así con una herramienta complementaria construida a través de una diversidad de librerías que ayudan al modelado 2D (dos dimensiones) y 3D (tres dimensiones) como lo son SDL (Simple DirectMedia Layer) y OpenGL (Open Graphics Library), y la facilidad con la que trabajan juntas.

Adicionalmente aplicar técnicas de renderizado como las tuberías, para la conversión de datos y obtener un resultado sensible a eventos del usuario; tomando en cuenta el cómo se visualizan los objetos en el espacio por algoritmos de transformaciones de objetos 3D (traslaciones, rotaciones y escalados).

Destacando su funcionamiento para temas tan importantes como lo son electrones de valencia y enlaces químicos, así como entender todo el comportamiento que conlleva.

\section{Metodología}

El proyecto se desarolló en dos periodos semestrales comprendidos entre los meses de Enero del 2018 a Enero del 2019, siendo la primera acción, la selección de Scrum como metodología de desarrollo, ya que permite abordar proyectos complejos, se basa en entregas parciales de manera regular y permite realizar el desarrollo con mayor rapidez. Esta metodología cuenta con tres roles principales: Project owner (propietario del proyecto) que define los objetivos y verifica que todo se realiza de manera correcta, Master Scrum (experto en Scrum) resuelve los problemas que surgen en el team (equipo) y el Scrum team (equipo Scrum) que es el equipo de desarrollo.

Esta metodología es definida por Laínez (2015) como ideal para el desarrollo incremental en escenarios complejos donde los requisitos tienen a cambiar frecuentemente. En la implementación de esta metodología se trabajó en fases, tales como: análisis, diseño, desarrollo y pruebas. 


\section{Análisis}

El proceso inició con la elaboración del "Product Backlog" (pila de producto). Se trata de un documento en que se recaba toda la información relevante acerca del proyecto como las tareas que se realizarán, los requerimientos a cumplir y todas las funcionalidades que el proyecto requiera. Para comenzar la recolección de requerimientos se realizaron continuas entrevistas con expertos en el tema para delimitar el alcance, enfoque, objetivos, impacto y aplicación del simulador, las características y comportamiento de las reacciones químicas a modelar.

A partir de lo anterior se determina el diseño de un sistema a la medida que sea capaz de elaborar de una manera rápida, eficiente y clara el modelado, representación, y simulación de reacciones químicas para así ayudar a la comprensión y entendimiento sobre cómo los elementos interactúan entre sí.

Después de contar con la información clasificada y categorizada por funcionalidades, se inició con la elaboración del documento de Especificación de Requerimientos de Software (ERS) para lo cual se utilizó la plantilla que ofrece el IEEE Std 830-1998 del Institute of Electrical and Electronics Engineers (IEEE, 1998), en este documento se especificaron los roles de los partipantes, las características de los usuarios, el alcance del software, así como los requerimientos funcionales y no funcionales. A continuación se muestra la descripción de algunos requerimientos específicos del ERS:

\begin{tabular}{|lll|l|}
\hline $\begin{array}{l}\text { Númeron de requisito } \\
\text { Nombre de requisito }\end{array}$ & $\begin{array}{l}\text { R1.11 } \\
\text { Mostrar simulación de cada elemento utilizando el } \\
\text { modelo Bohr }\end{array}$ \\
\hline $\begin{array}{l}\text { Tipo } \\
\text { Fuente del requisito } \\
\begin{array}{l}\text { Prioridad del } \\
\text { requisito }\end{array}\end{array}$ & $\begin{array}{l}\text { Entrequisito } \\
\text { Revista } 28 / 03 / 2018, \text { registro 12 }\end{array}$ \\
\hline
\end{tabular}

\begin{tabular}{|c|c|c|c|}
\hline Número de requisito & \multicolumn{3}{|l|}{ R1.13 } \\
\hline Nombre de requisito & \multicolumn{3}{|c|}{$\begin{array}{l}\text { Crear módulo de reconocimiento óptico de } \\
\text { compuestos }\end{array}$} \\
\hline Tipo & $\triangle$ Requisito & $\square$ Restricción & \\
\hline Fuente del requisito & \multicolumn{3}{|c|}{ Entrevista $28 / 03 / 2018$, registro 12} \\
\hline $\begin{array}{l}\text { Prioridad del } \\
\text { requisito }\end{array}$ & 凶 Alta/Esencial & $\square$ Media/Deseado & $\square$ Baja \\
\hline
\end{tabular}

\begin{tabular}{|c|c|c|c|}
\hline \multirow{3}{*}{$\begin{array}{l}\text { Número de requisito } \\
\text { Nombre de requisito } \\
\text { Tipo }\end{array}$} & \multicolumn{3}{|l|}{ R1.14 } \\
\hline & \multicolumn{3}{|c|}{ Resaltar elementos de acuerdo a su clasificación } \\
\hline & \ Requisito & $\square$ Restricción & \\
\hline Fuente del requisito & \multicolumn{3}{|c|}{ Entrevista 28/03/2018, registro 12} \\
\hline $\begin{array}{l}\text { Prioridad del } \\
\text { requisito }\end{array}$ & \Alta/Esencial & $\square$ Media/Deseado & $\square$ Baja \\
\hline
\end{tabular}

Tabla 1 Especificación de requerimientos Fuente: Elaboración Propia
La segunda etapa es la definición del Sprint Backlog (lista de tareas), documento en que se definen las tareas que se deben de realizar $y$ se asigna a la persona encargada de desarrollarlas, además se asigna el periodo en el que se deberá realizar cada una de las actividades, para esta tarea se realizó un diagrama de Gantt.

Se denomina Sprint a un periodo determinado de tiempo en el que se realizan las acciones que fueron descritas durante el Sprint Backlog, El objetivo de los sprints es obtener entregas parciales que permitan realizar pruebas.

En esta etapa diariamente se realizan juntas con los miembros del equipo con una duración no mayor a 15 minutos, en estas reuniones mejor conocidas con Daily Scrum (reunión diaria) se habla acerca de lo que se ha hecho, lo que se planea hacer y los problemas o impedimentos que se tuvieron.

\section{Diseño}

La primera tarea en el diseño arquitectónico fue el desarrollo del diagrama de bloques, que permitió realizar un bosquejo de los módulos o fragmentos de la funcionalidad y/o modelos de la aplicación, una vez definidos se continuó con el diagrama de clases para ofrecer mayor nivel de detalle, así como determinar todos los fragmentos auxiliares necesarios para el funcionamiento; mostrando las clases que componen el sistema describiendo sus atributos, métodos y relaciones pertinentes.

Se realizó el diagrama de casos de usos para modelar los escenarios en los cuales el usuario interactúa con el sistema y entender el cómo es que acceden a las distintas funcionalidades, así como ver las relaciones que existen entre los casos de usos planteados.

En cuanto al modelado de datos se realizó el diagrama relacional para definir de manera clara y concisa la estructura de la base de datos, con el objetivo de describir su contenido. A continuación se presenta el modelo relacional: 


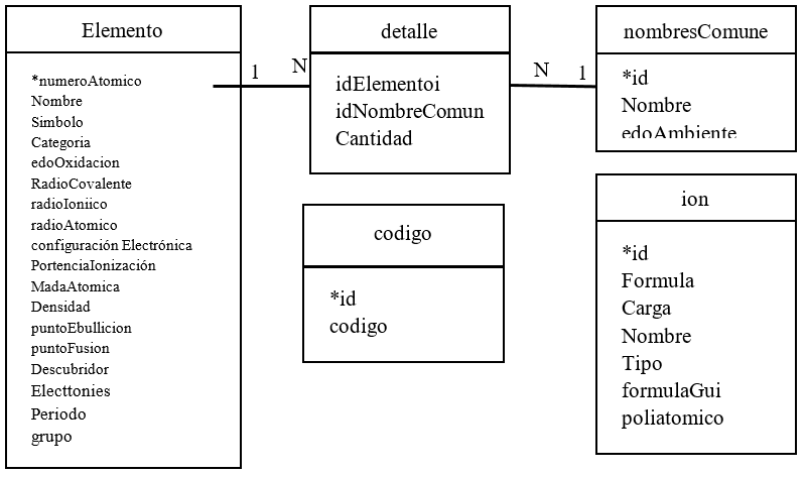

Figura 1 Modelo relacional Fuente: Elaboración Propia

En este modelado de datos, se implementó también la definición de los metadatos que conformarán la estructura de almacenamiento de la Base de Datos.

\section{Desarrollo aplicación móvil}

Esta aplicación es complementaria a la aplicación de escritorio, específicamente para el sub-módulo de reconocimiento óptico de compuestos.

Se pretende que cualquier usuario a través de un dispositivo móvil pueda tomar fotos de cualquier compuesto escrito a mano (por ejemplo después de una clase) para después de ciertas transformaciones y filtros que se realizan a la imagen se envíen a un servidor, donde la aplicación principal tendrá un hilo de ejecución trabajando en todo momento en busca de imágenes por reconocer, $\mathrm{y}$ así hacer su respectiva simulación. A continuación se muestra la interfaz de inicio, desde la cual se realiza la toma de fotografía:

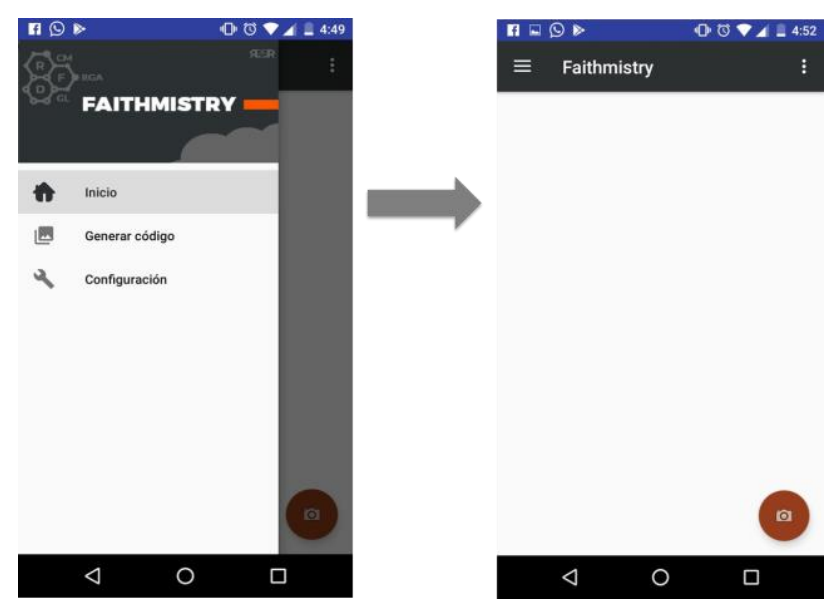

Figura 2 Interfaz de inicio móvil Fuente: Elaboración Propia
En la aplicación móvil se desarrolló la funcionalidad que permitirá la configuración del servidor al que se desea conectar, y el cual contará con toda la funcionalidad de creación de directorios dinámicos.

\section{Desarrollo aplicación web}

Esta aplicación esta destinada a la administración de los registros en la Base de Datos, permitiendo realizar operaciones CRUD (create, read, update and delete), actualizando los datos relacionados con los elementos de la tabla periódica, ya sea por alguna actualización derivada de algun descubrimiento o como proceso de corrección de algún dato ingresado incorrectamente. A continuación se muestra la interfaz que permite visualizar los iones:

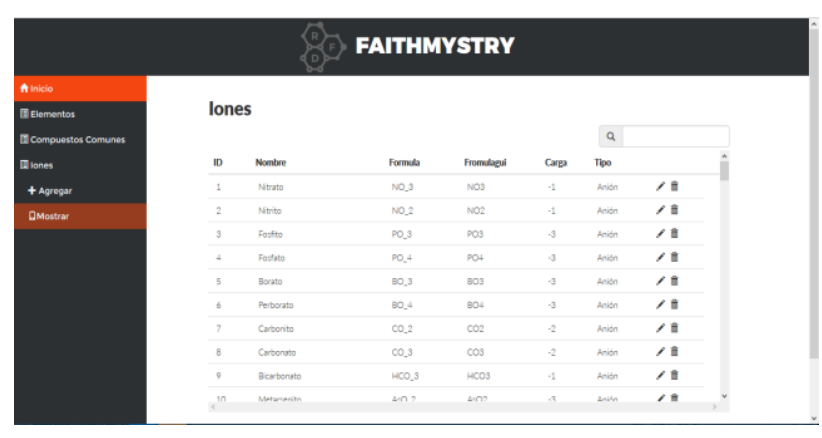

Figura 3 Interfaz de visualizar iones

Fuente: Elaboración Propia

\section{Desarrollo aplicación de escritorio}

Esta es la aplicación principal, en donde se encuentra la mayoría de las funcionalidades del sistema, dentro de ella se encuentran distintos módulos, tales como la tabla periodica, simulación de elementos y síntesis.

\section{Módulo tabla periódica}

Dinámica e interactiva, con el objetivo de conocer con un alto nivel de detalle $y$ antecedentes los elementos que la conforman, así como conocer la estructura de cada átomo a nivel subatómico.

Los elementos son cargados en un vector, obtenidos de la base de datos con el uso de sockets de red. Este vector será usado para procesar los objetos y sus respectivos eventos. La interfaz de la tabla periódica fue creada en SDL, y cada elemento en pantalla es dibujado uno por uno posicionando en un eje coordenado de 2 dimensiones. 


\section{Módulo de simulación de elementos}

Este módulo es el núcleo del software, encargado del cálculo, modelado, representación, simulación y respectiva animación 3D de reacciones químicas a través de la selección de elementos de la tabla periódica, compuestos pre-definidos, y/o iones. Interactuando de manera amigable con átomos y moléculas para conocer así su estructura, bajo el umbral de la estequiometría. Las animaciones fueron realizadas utilizando openGL para $\mathrm{C}++$.

la aplicación de escritorio se pueden diferenciar dos tipos de simulaciones: simulación de elementos y simulación de compuestos. A continuación se muestra el pseudocódigo de transformación de coordenadas esféricas a rectangulares y el uso de la ecuación paramétrica de la circunferencia

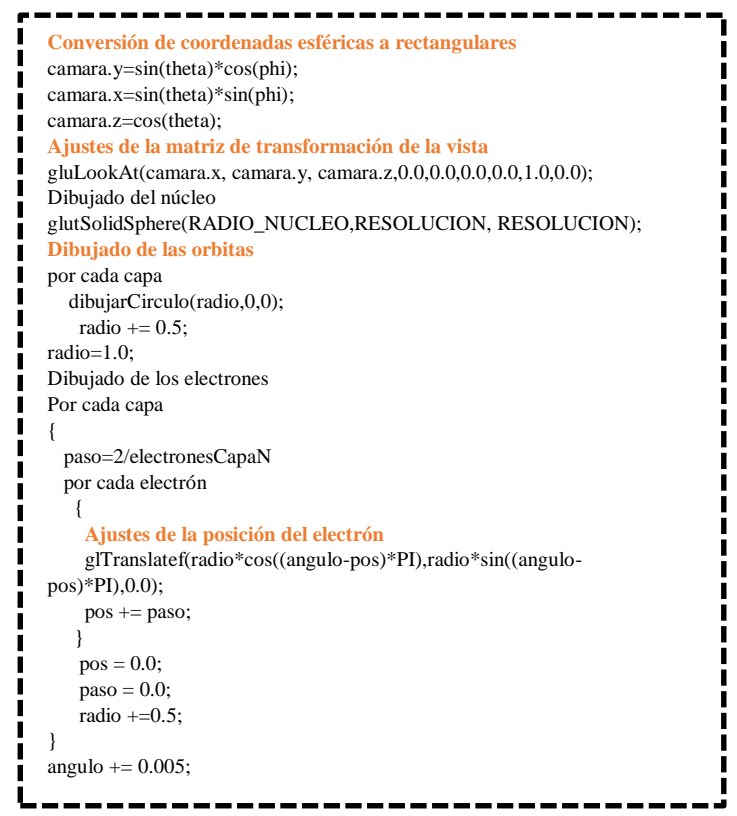

Figura 4 Pseudocódigo de transformación de coordenadas esféricas a rectangulares y el uso de la ecuación paramétrica de la circunferencia

Fuente: Elaboración Propia

Debido a que openGL carece de primitivas para realizar círculos, deben dibujarse haciendo uso de un arreglo de 360 vértices, uno por cada grado, para formar este polígono, se hace uso de la ecuación paramétrica de la circunferencia para determinar la posición de cada uno de los vértices en sus 360 grados. En cuanto a la simulación de compuestos se optó por el uso de un analizador sintáctico para la lectura de los compuestos ingresados por el usuario debido a que asegura que el compuesto fue ingresado correctamente y la formación del árbol sintáctico es la adecuada para facilitar la determinación del orden de simulación.

\section{Núcleo de simulación y grafo de valencias}

El núcleo de simulación es el encargado de comprobar si es posible la unión de un par de elementos, así como de determinar el tipo de compuesto generado a partir de dicha unión; ese proceso está implementado mediante una máquina de estados, en la cual los tipos de compuestos son determinados a partir de la categoría de los elementos que se desea unir. Su desarrollo al igual que cualquier máquina de estados fue implementada mediante variables específicas dentro de la clase núcleo. A continuación se muestra la representación de la máquina de estados:

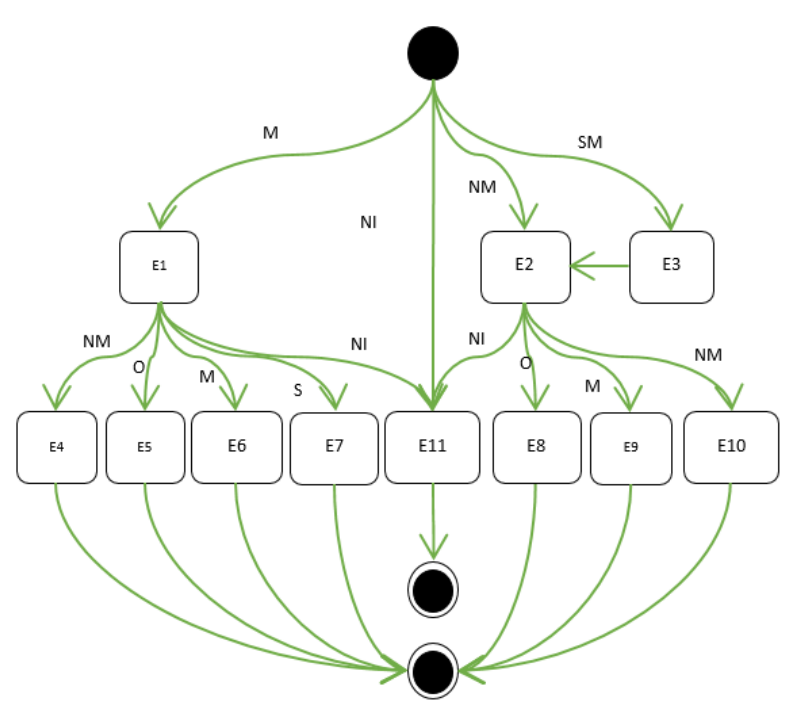

Figura 5 Máquina de estados para compuestos compatibles

Fuente: Elaboración propia

Una vez que se ha comprobado que el compuesto es posible, se determinan las valencias con las que trabajan los elementos que conforman el compuesto, pues determinan las cargas parciales de los subproductos del compuesto, además de ser un apoyo vital para determinar la configuración final y las cargas con las que trabajarán cada una de sus uniones.

El grafo ponderado es construido utilizando los propios elementos como nodos y sus valencias como pesos para las aristas. A continuación se muestra el grafo de elementos para la formación de compuestos y valencias compatibles:
MACÍAS-BRAMBILA, Hassem Rubén, PULIDO-GONZÁLEZ, Héctor, GÓMEZ-LÓPEZ, Daniela y GUTIÉRREZ-ÁLVAREZ, Francisco Ramón. Modelado e implementación de un sistema distribuido para la simulación de reacciones químicas. Revista de Aplicaciones de la Ingeniería. 2019. 


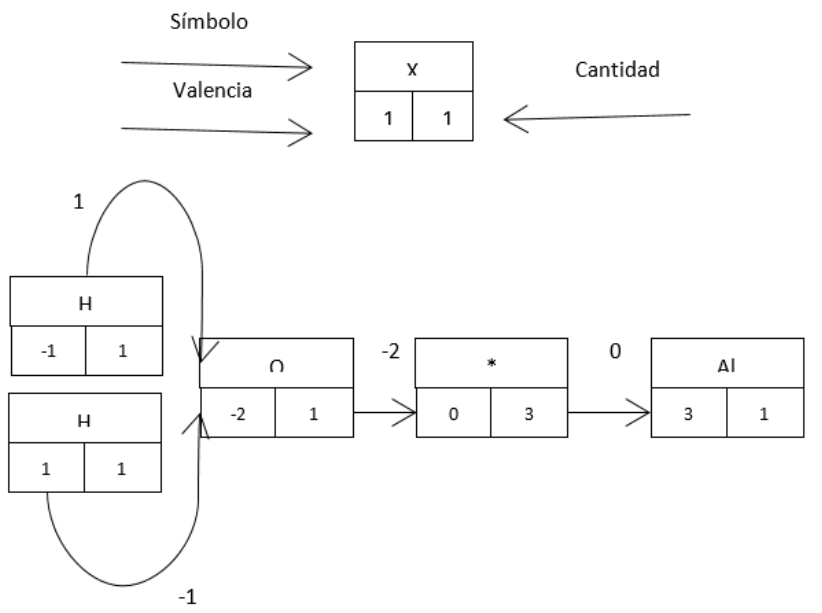

Figura 6 Grafo de elementos para la formación de compuestos y valencias compatibles

Fuente: Elaboración Propia

\section{Síntesis}

La funcionalidad de simulación de reacciones requiere que el usuario seleccione los compuestos que estarán involucrados en cada una de ellas, después de este proceso es posible visualizar su nombre y la fórmula, lo que inicia el proceso, solo presionando la barra espaciadora. A continuación se muestra una imagen con el compuesto Bromuro de aluminio representado por la fórmula $\mathrm{AlBr}_{3}$ :

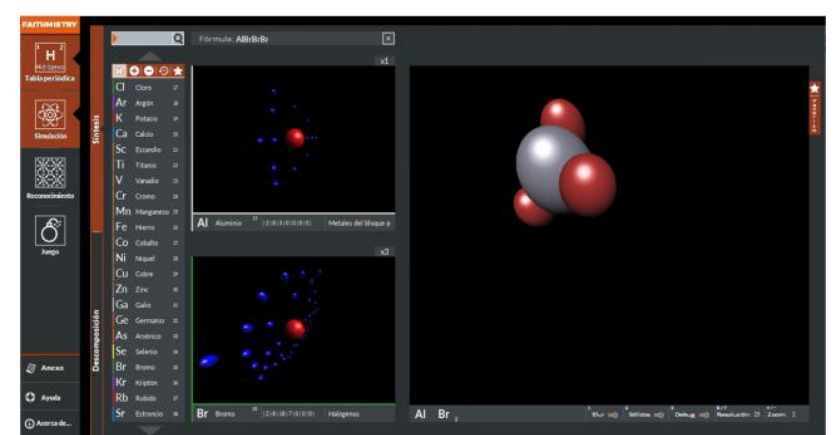

Figura 7 Simulación de reacción del compuesto $\mathrm{ALBr}_{3}$. Fuente: Elaboración Propia

A continuación se muestra una imagen con la simulación de dióxido de carbono $\mathrm{CO}_{2}$, se seleccionó de la lista de elementos, dos átomos de oxígeno y un átomo de carbono.

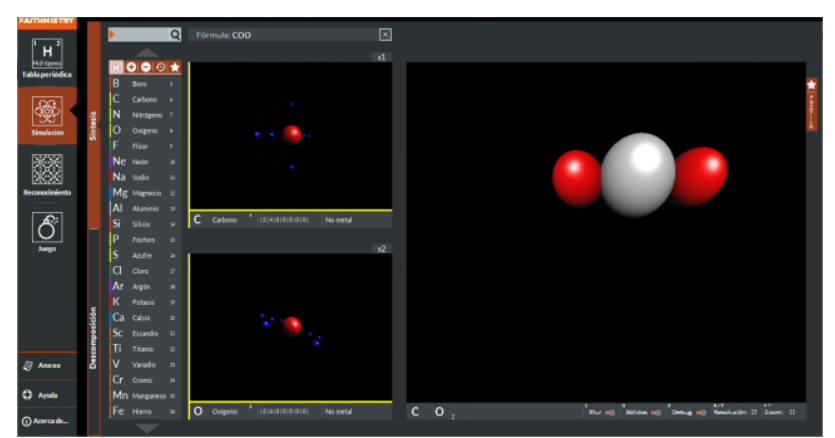

Figura 8 Simulación de reacción del compuesto $\mathrm{CO}_{2}$. Fuente: Elaboración Propia
En la siguiente imagen se puede observar la simulación de $\mathrm{AsO}_{2}$, para esta simulación se seleccionó de la lista de aniones el compuesto Metarsenito.

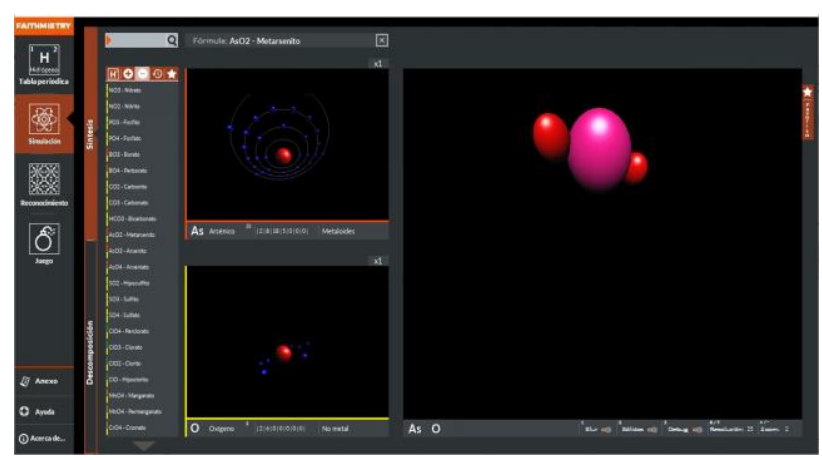

Figura 9 Simulación de reacción del anión $\mathrm{AsO}_{2}$ Metarsenito

Fuente: Elaboración Propia

Otra forma de realizar simulaciones es a través de la captura de la fórmula del compuesto, en ella se pueden incluir varias moléculas del mismo compuesto, por ejemplo en la siguiente imagen se encuentran 5 moléculas del anión de Borato.

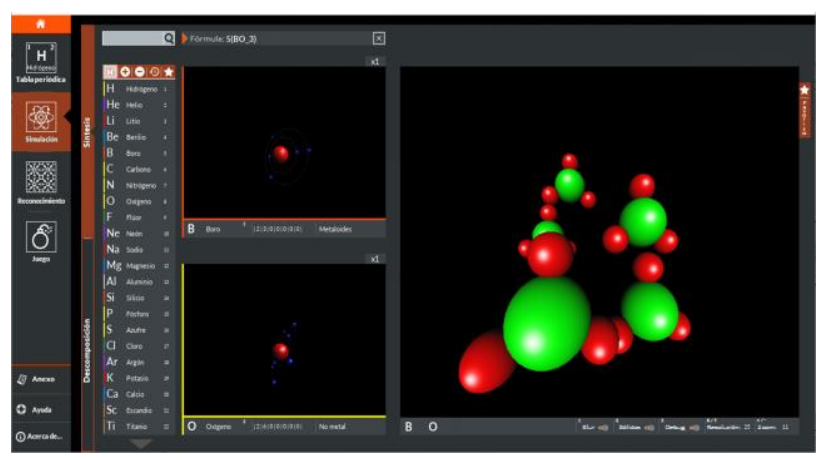

Figura 10 Simulación de reacción de 5 moléculas del anión Borato

Fuente: Elaboración Propia

\section{Pruebas}

En esta fase se diseñó e implementó un plan de pruebas para las aplicaciones y la configuración e instalación de los servidores que almacena la base de datos, la aplicación web y el procesamiento de datos. Este plan de pruebas contempla información general del proyecto, así como un historial de versiones y la definición del alcance de las pruebas, el cual incluyó:

- Elementos de pruebas

- Funcionalidades a probar

- $\quad$ Pruebas de regresión

- Funcionalidades a no probar

- $\quad$ Estrategia de pruebas

MACÍAS-BRAMBILA, Hassem Rubén, PULIDO-GONZÁLEZ, Héctor, GÓMEZ-LÓPEZ, Daniela y GUTIÉRREZ-ÁLVAREZ, Francisco Ramón. Modelado e implementación de un sistema distribuido para la simulación de reacciones químicas. Revista de Aplicaciones de la Ingeniería. 2019. 
Además; con el objetivo de determinar un desempeño aproximado de la ejecución de los Casos de Uso, se definieron a través de los requerimientos funcionales y no funcionales, los criterios de aceptación y rechazo de las pruebas a través de un comportamiento binario. Así como la determinación de los entregables, los recursos, la planeación y la organización para la ejecución del plan de pruebas.

\section{Resultados}

Se determinó establecer un Sistema distribuido a través de un modelo cliente-servidor, que además cuenta con una suite de aplicaciones que realizan peticiones al servidor de datos y de procesamiento, de tal manera que el dispositivo móvil no requiera con sus recursos limitados el procesamiento de la imagen para la determinación del compuesto con el reconocimiento de patrones.

En cuanto al almacenamiento de datos, por su baja complejidad se determino establecer un modelo centralizado de datos para su administración debido a que la estimación realizada de acuerdo a la capacidad operativa de una jornada en un laboratorio de química no contempla una conectividad simultánea de más de 40 usuarios, por lo que las peticiones de los clientes, la gestión del almacenamiento de datos y del procesamiento de operaciones, no justifican el costo de una base de datos distribuida.

En cuanto a la determinación del entorno de desarrollo, de instalación y configuración del servidor no se contempló ningún tipo de restricción salvo la adquisición de licencias, por lo que se optó por la implementación de tecnologías libres, lo que favorece la adaptación de las tecnologías a sus procesos por no representar costos de licenciamiento.

Se realizaron a través del plan de pruebas, casos que contemplaron aspectos de conectividad, rendimiento, interfaz y funcionalidad; para los cuales se comprobó efectivamente la conexión simultánea de 135 usuarios y sus respectivas peticiones como clientes, así como las salidas de los procesos, con estadística descriptiva se determina la coincidencia en un $97.75 \%$ con los resultados esperados en los casos de prueba. El resto de las salidas que no cumplieron los criterios de aceptación se documentaron y se reprocesaron.

\section{Conclusiones}

El ser humano está en contacto directo con cambios químicos, muchos de los cuales dependen de actividades diarias e incluso de nuestra vida. Los hallazgos y avances que se han tenido acerca de las fórmulas y las leyes que rigen las combinaciones ha permitido tener mejores condiciones en diversos ámbitos. Todo ésto basado en entender el concepto completo de las reacciones químicas y cuán importantes son, por ello en la actualidad resulta muy importante tener y comprender cómo estos cambios ocurren. Así como el contar con herramientas accesibles que logren abarcar el concepto fundamental y ayude a clarificar tópicos como lo son electrones de valencia y enlaces químicos.

A lo largo del presente proyecto se demuestra con éxito la capacidad de aplicar y aprovechar la tecnología enfocada a la estequiometría, una parte de la química.

Implementando así, diversos desarrollos, módulos, instrumentos de aplicación y mecanismos para recolección y validación de datos acerca del comportamiento de las reacciones químicas. Consultando con expertos en el área en diferentes sesiones de trabajo para establecer los modelos de comportamiento de las reacciones químicas a representar y consolidando bases sólidas, generando así diferentes modelados de diseño para la correcta representación de la aplicación.

Se estableció la importancia de la inteligencia artificial y, específicamente, las redes neuronales, siendo útiles en aplicaciones de reconocimiento de patrones, presentando gran flexibilidad y la posibilidad de ser escaladas a un sin número de situaciones y problemáticas.

Una observación detallada conduce a una mejor comprensión del sistema y su operación. Se tiene un mejor entendimiento cómo es la interacción directa entre elementos de la tabla periódica, iones y compuestos seleccionados, ayudando así, a vislumbrar las variables más importantes que interactúan en el sistema y a entender mejor las interrelaciones entre estas variables. 
Además se destaca la importante labor de crear simulaciones en ambientes tridimensionales actualmente, ya que se pueden establecer entornos detallados para estudiar efectos de cambios internos y externos de un sistema completo al hacer alteraciones en el modelo de un sistema y observando los efectos resultantes.

La técnica de simulación ayuda a experimentar con nuevas situaciones, sobre las cuales se tiene poca o ninguna información; anticipando lo mejor posible a resultados no previstos.

Generando entonces, un canon para el desarrollo de futuros proyectos bajo el umbral de la estequiometría, con la intención de añadir más módulos como reacciones de desplazamiento ya sea simple o doble según su estructura, incluso reacciones de acuerdo a la energía que es intercambiada como calor, luz y energía eléctrica, lo que permitirá ampliar el alcance del proyecto, impactando en una cantidad mayor de asignaturas y Programas Educativos que requieren en su mapa curricular el estudio de reacciones químicas.

\section{Referencias}

Andrea Rueda (2006). Introducción a 3D Transformaciones 3D, Introducción a la Computación Gráfica. Universidad Javeriana, Depto. de Ingeniería de Sistemas.

BITSAdmin Tool Recuperado el 25 de
septiembre de
https://msdn.microsoft.com/en-
us/library/windows/desktop/aa362813(v=vs.85)
.aspx

Bracho J, El plano euclidiano. Recuperado el día 15 de agosto de 2017 de: http://www.matem.unam.mx/ rgomez/geometri a/Capitulo1.pdf.

Chang, R. (2017). Química General. (7 Ed). McGraw-Hill.

Corey, R. B., \& Pauling, L. (1953). Molecular models of amino acids, peptides, and proteins. Review of Scientific Instruments, 24(8), 621627.
Cortez Roberto (2002) Introducción Al Análisis de Sistemas Y la Ingeniería de Software. Editorial Universidad Estatal a Distancia.

D. Hearn, M.P. Baker (2011). Gráficos por computadora con OpenGL, 3a edición. Pearson Prentice Hall.

Didact S,L (2005) Manual de programación Lenguaje C++ Primera edición Editorial MAD.

Escolano, F., Cazoria, M., Pardo, M. G., \& Lozano, M. (2006). Inteligencia Artificial . Thomson.

Flórez R, Fernández J.M. (2008). Las Redes Neuronales Artificiales. Netbiblio, S.L.

García González, C. A., Ayala Armijos, J., \& Martínez Mora, O. (2015). Química inorgánica.

Gillespie R., Hargittai I., (2012) The VSEPR model of molecular geometry Ed. Dover.

Grupos y periodos Recuperado el 19 de agosto de 2017 de: http://recursostic.educacion.es/secundaria/edad/ 4esofisicaquimica/4quincena8/4q8_contenidos_ 3c_ampliacion.htm.

Hargittai, I., \& Chamberland, B. (1986). The VSEPR model of molecular geometry. In Symmetry (pp. 1021-1038).

Hearn-Baker (2013). Gráficos por computadora con OpenGL. Tercera edición, Indiana University, PEARSON Prentice Hall.

Hernández, F. G., Granados, A. P., \& Gallegos, S. R. (2016). Química I. Santillana.

IUPAC. Compendium of Chemical Terminology, 2nd ed. (the "Gold Book"). Compiled by A. D. McNaught and A. Wilkinson. Blackwell Scientific Publications, Oxford (1997).

IEEE. (1998). IEEE Std 830-1998 - IEEE Recommended Practice for Software Requirements Specifications. mayo 20, 2017, de C - IEEE Computer Society Sitio web: https://standards.ieee.org/findstds/standard/8301998.html. 
Izquierdo, J. F., Cunil, F., Tejero, J., Iborra, M., \& Fité, C. (2004). Cinética de las Reacciones Químicas. Edicions de la Universitat de Barcelona.

J.D. Foley, A. van Dam, S.K. Feiner, J.F. Hughes (1996). Computer graphics: principles and practice, 2nd edition in C. Addison-Wesley.

Kenneth Connors (1990). Chemical Kinetics, VCH Publishers.

Kernighan B.W, Ritchie M.D.(2015). The C Programing Lenguage. (2 ed.) Pearson.

Koltun, W. L. (1965). U.S. Patent No. 3,170,246. Washington, DC: U.S. Patent and Trademark Office.

Klein, C., \& Hurlbut, C. S. (1996). Manual de mineralogía (Vol. 2). Reverté.

Kotz, J. C., Treichel, P. M., \& Townsend, J. R. (2009). Chemistry \& Chemical Reactivity. Thomson Books /Cole.

Laínez Fuentes José Rubén (2015) Desarrollo de Software Ágil Extremme Programming y Scrum (2nd edition) Ed. IT Campus Academy.

Leigh, G. J. (Ed.). (1990). Nomenclature of inorganic chemistry: recommendations 1990. Oxford: Blackwell scientific publications.

León, A., Daniel, J., \& Tabares Hurtado, J. D. (2019). Desarrollo de un simulador de control automático de procesos en Microsoft Excel para ser utilizado en el laboratorio virtual de control y simulación de procesos.

Morales, J. P., Hurtado, J. I., Mejía, A. T., \& Cuartas, J. H. (2019). Metodología de Ajuste de un NMPC con Sistema de Inferencia Borrosa Takagi Sugeno y Conjuntos Borrosos Multidimensionales para Aplicaciones en Procesos Químicos no Lineales. Revista beroamericana de Automática e Informática industrial, 16(1), 100-113.

Morris Hein y Arena Susan (2014). Foundations of collage chemistry (14 ed.). John Wiley \& Sons Inc.
Muñoz, J. C. M. (2014) Aplicación de una estrategia didáctica que permita la comprensión de la estequiometría a partir de un aprendizaje significativo. Universidad Nacional de Colombia.

Parry Robert (1987). Chemistry, Experimental Foundations, Prentice-Hall, Inc.

Pilone, D., Pitman, N. (2009). UML 2.0 in a Nutshell. O’Reilly Media, Inc.

Ríos, A. (2019). Implementación de un modelo hidrodinámico tridimensional en el embalse de Paso Severino. Aportes para la modelación de calidad de agua.

Romero J. Especificación de Requisitos Software según el estándar IEEE 830. Recuperado el día 8 de agosto de 2017 de: https://www.fdi.ucm.es/profesor/gmendez/docs/ is0809/ieee830.pdf

Russell, S., \& Norving, P. (2004). Inteligencia Artificial: Un enfoque moderno . Pearson.

San Fabián, J. Aguado, A. y de Ema, I., Estequiometria. Recuperado el día 15 de agosto de 2017 de: http://www.qfa.uam.es/qq/tema1i.pdf

Scott, F. H. (2008). Elementos de Ingeniería de las Reacciones Químicas. (cuarta, Ed.) Mexico: Person Educación .

Sommerville, I. (2015). Software Engineering (10 ed.). Person. 